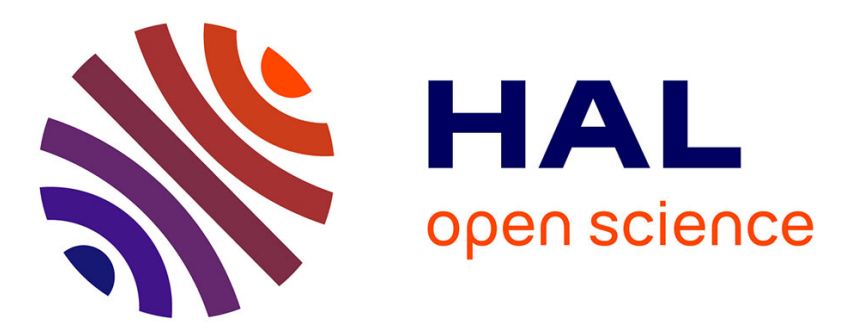

\title{
Structure of electron and ion distribution functions near a reconnection line
}

\author{
Roch Smets, D. Delcourt, D. Fontaine, R. Martin
}

\section{To cite this version:}

Roch Smets, D. Delcourt, D. Fontaine, R. Martin. Structure of electron and ion distribution functions near a reconnection line. Journal of Geophysical Research Space Physics, 1996, 101 (A11), pp.2483724845. 10.1029/96JA02179 . hal-02994641

\section{HAL Id: hal-02994641 \\ https://hal.science/hal-02994641}

Submitted on 8 Nov 2020

HAL is a multi-disciplinary open access archive for the deposit and dissemination of scientific research documents, whether they are published or not. The documents may come from teaching and research institutions in France or abroad, or from public or private research centers.
L'archive ouverte pluridisciplinaire HAL, est destinée au dépôt et à la diffusion de documents scientifiques de niveau recherche, publiés ou non, émanant des établissements d'enseignement et de recherche français ou étrangers, des laboratoires publics ou privés. 


\title{
Structure of electron and ion distribution functions near a reconnection line
}

\author{
R. Smets and D. Delcourt \\ Centre d'étude des Environnements Terrestre et Planétaires, Centre National de la Recherche \\ Scientifique, Saint-Maur des Fossés, France \\ D. Fontaine \\ Centre d'étude des Environnements Terrestre et Planétaires, Centre National de la Recherche \\ Scientifique, Vélizy, France \\ R. F. Martin Jr. \\ Physics Department, Illinois State University, Normal
}

\begin{abstract}
We examine the transport of electrons and ions in the vicinity of an $\mathrm{X}$ line in the magnetotail, using single-particle trajectory codes. It is shown that the particle distribution functions downstream of the reconnection region exhibit two distinct density domains separated by a narrow channel of enhanced densities. This narrow structure, which was first reported for ions by Martin and Speiser [1988] and referred to as a ridge, is due to particles which are essentially $\mathbf{E} \times \mathbf{B}$ convected from the lobes and experience large energy gains upon interaction with the neutral line. This structure weakly depends upon the characteristics of the inflowing population. As for the density domains on either side, they result from mixing of the particle populations during the reconnection process, high and low densities corresponding to particles originating earthward and tailward from the neutral line, respectively. The simulations also reveal fine low-density structures embedded ir. the overall pattern. It is shown that these fine structures result from prominent gradient drift effects upon approach of the field reversal. It is demonstrated that, at a given observation point downstream of the reconnection region, the computed distribution functions strongly depend upon the azimuthal direction of view. An analytical estimate is proposed for the ridge location, which provides information on the neutral line characteristics.
\end{abstract}

\section{Introduction}

Particles traveling in the distant magnetotail do not conserve their magnetic moment (first adiabatic invariant) because of significant field variations on the length scale of their cyclotron turn. In a one-dimensional $(X-$ and $Y$-invariant) field geometry, as is nearly the case in the distant magnetotail, the nonadiabatic particle behavior can be organized by using the $\kappa$ parameter defined as the square root of the minimum curvature radius to maximum Larmor radius ratio [e.g., Büchner and Zelenyi, 1989]. For $\kappa>3$ the particle motion is adiabatic (magnetic moment conserving), and the guiding center approximation is valid. For $\kappa \simeq 1$ the motion may be chaotic, and three distinct regimes of magnetic moment variations are obtained depending upon pitch angle [e.g., Delcourt et al., 1995]. For $\kappa<1$ with the

Copyright 1996 by the American Geophysical Union.

Paper number 96JA02179.

0148-0227/96/96JA-02179\$09.00
Larmor radii becoming larger than the field variation length scale, particles can experience fast oscillations (meandering motion) about the field minimum as was initially shown by Speiser [1965]. In this case, various classes of particle orbits have been identified (transient or Speiser-type, stochastic, trapped) which occupy distinct regions of the phase space [e.g., Chen and Palmadesso 1986; Chen, 1992].

Such an interpretation framework based upon the $\kappa$ parameter is not appropriate in a two-dimensional field geometry such as near the $\mathrm{X}$ line, which forms in the far tail when the interplanetary magnetic field (IMF) is southward. Several studies have been dedicated to the analysis of particle motion under such conditions, using, for instance, Lyapunov exponent calculations [Martin, 1986] or Poincaré surfaces of section [Burkhart et al., 1991]. Here the issue of primary interest is to identify characteristic signatures associated with the reconnection process, which is otherwise inaccessible to observations because of its localized character. One of the consequences of reconnection in the far tail is a large 
acceleration of ions and the production of ion beams [e.g., Curran and Goertz, 1989], which yield characteristic earthward streaming populations in the plasma sheet boundary layer (PSBL) [e.g., Zelenyi et al., 1990; Onsager et al., 1991]. In the plasma sheet, downstream of the reconnection region, such beams may also form as a result of Speiser-type motion at specific "resonant" energies [e.g., Burkhart and Chen, 1991]. On the other hand, Martin and Speiser [1988] demonstrated that nonadiabatic motion near the $\mathrm{X}$ line leads to a split of ion distribution functions into two distinct density regions. Doxas et al. [1994] showed that abrupt density and temperature changes should occur near the neutral line. Joyce et al. [1995] found that low- and high-energy ion populations coexist in the reconnection region, the relative importance of which depends upon elevation in the field reversal.

In this study we revisit the result of Martin and Speiser [1988] and examine in particular its consequences for electron distribution functions. Because of their much smaller Larmor radii, the motion of electrons turns nonadiabatic in a region of space much smaller (likely, the immediate vicinity of the reconnection line) than that obtained for ions [e.g., Lyons, 1984]. Schriver and Ashour-Abdalla [1993] showed that interaction with the X line at $\simeq 100 R_{E}$ in the Tsyganenko [1989] model leads to the formation of electron beams as well as to an embryonic plasma sheet downstream of the neutral point. These authors, however, did not address the full three-dimensional structure of the distribution function at a given point of observation. In the following, we will first investigate the overall structure of electron and ion distributions and discuss its variability with initial conditions in the magnetospheric lobes. We will show that there exist fine structures superimposed onto the overall distribution pattern and due to gradient drift effects upon approach of the field reversal. Finally, we will discuss information that can be gained from these results with regard to the reconnection process, and we will present an analytical description of the distribution function characteristics.

\section{Model Distribution Functions}

To investigate the structure of distribution functions near a reconnection line, we adopted a magnetic field model similar to that used by Martin and Speiser [1988], namely,

$$
\mathbf{B}=-b_{0} z \mathbf{x}-b_{0} \delta x \mathbf{z}
$$

Here $\delta$ is a parameter controlling the angle between the magnetic field line separatrices in the reconnection region, and $\mathbf{x}$ and $\mathbf{z}$ are unit vectors in the $x$ and $z$ directions, respectively (with $x$ and $z$ in units of Earth radii). In this model the neutral point lies at the axis origin, and the magnetic field lines are hyperbolas such that $z^{2}-\delta \times x^{2}=$ const. In the following, we use the following parameter values: $b_{0}=12 \mathrm{nT} / R_{E}$, and $\delta=2.5 \times 10^{-3}$. Figure 1 shows the magnetic field

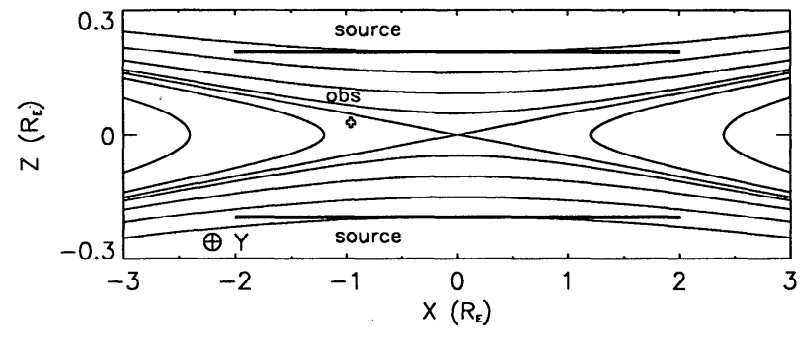

Figure 1. Magnetic field model used in the trajectory calculations (equation (1)). Heavy solid lines show the source location in the magnetospheric lobes, whereas the cross schematically shows the observation point.

lines thus obtained, with the $x$ axis pointing toward the tail and the $y$ axis pointing from dusk to dawn. The field line geometry in this figure closely resembles that in the Tsyganenko [1989] model $(K p=1-, 1,1+)$ at about $100 R_{E}$ in the geomagnetic tail, with similar field gradients in the $x$ and $z$ directions and an angle between separatrices earthward of the neutral point of the order of $6^{\circ}$. As for the electric field, we considered a uniform dawn-to-dusk (i.e., in the $-y$ direction in Figure 1) convection electric field of $0.2 \mathrm{mV} / \mathrm{m}$. To compute electron and ion distribution functions, we performed systematic trajectory calculations backward in time (using the full equation of motion integrated by means of a fourth-order Runge-Kutta technique) from a given observation point (cross in Figure 1) until the particles reach a source in the magnetospheric lobes intended to model inflowing plasma mantle populations. In the computations this source was placed at a given $z_{\text {source }}$ height on either side of the reconnection line (heavy solid lines in Figure 1) and taken as a drifting Maxwellian with a temperature of $200 \mathrm{eV}$, a bulk flow speed of $400 \mathrm{~km} \mathrm{~s}^{-1}$, and a density of $4 \times 10^{4} \mathrm{~m}^{-3}$. Once the test particles were weighted within the initial distribution, application of the Liouville theorem allowed us to reconstruct the distribution function at the observation point.

Figure 2 shows an example of the results obtained for electrons downstream of the reconnection site at $x_{o b s}=$ $-1 R_{E}$ and $z_{o b s}=0.01 R_{E}$ (with line sources placed at $z_{\text {source }}= \pm 0.2 R_{E}$ ). In the top panel of this figure an overall structure clearly emerges with a narrow channel of enhanced densities separating two distinct density regions. Simultaneously, in the bottom panel of Figure 2 , which depicts the electron time of flight from the source location until the observation point, it can be seen that this narrow channel coincides with relatively large times of flight (of the order of 2 to $4 \mathrm{~s}$ ). Moreover, note the skewing of this time-of-flight structure toward large pitch angles from low to high energies.

Ion distribution functions near the reconnection line exhibit a somewhat similar pattern. This is illustrated in Figure 3, which relates to protons with identical initial conditions in the magnetospheric lobes but with a temperature of $400 \mathrm{eV}$ and a line source at $z_{\text {source }}=$ 


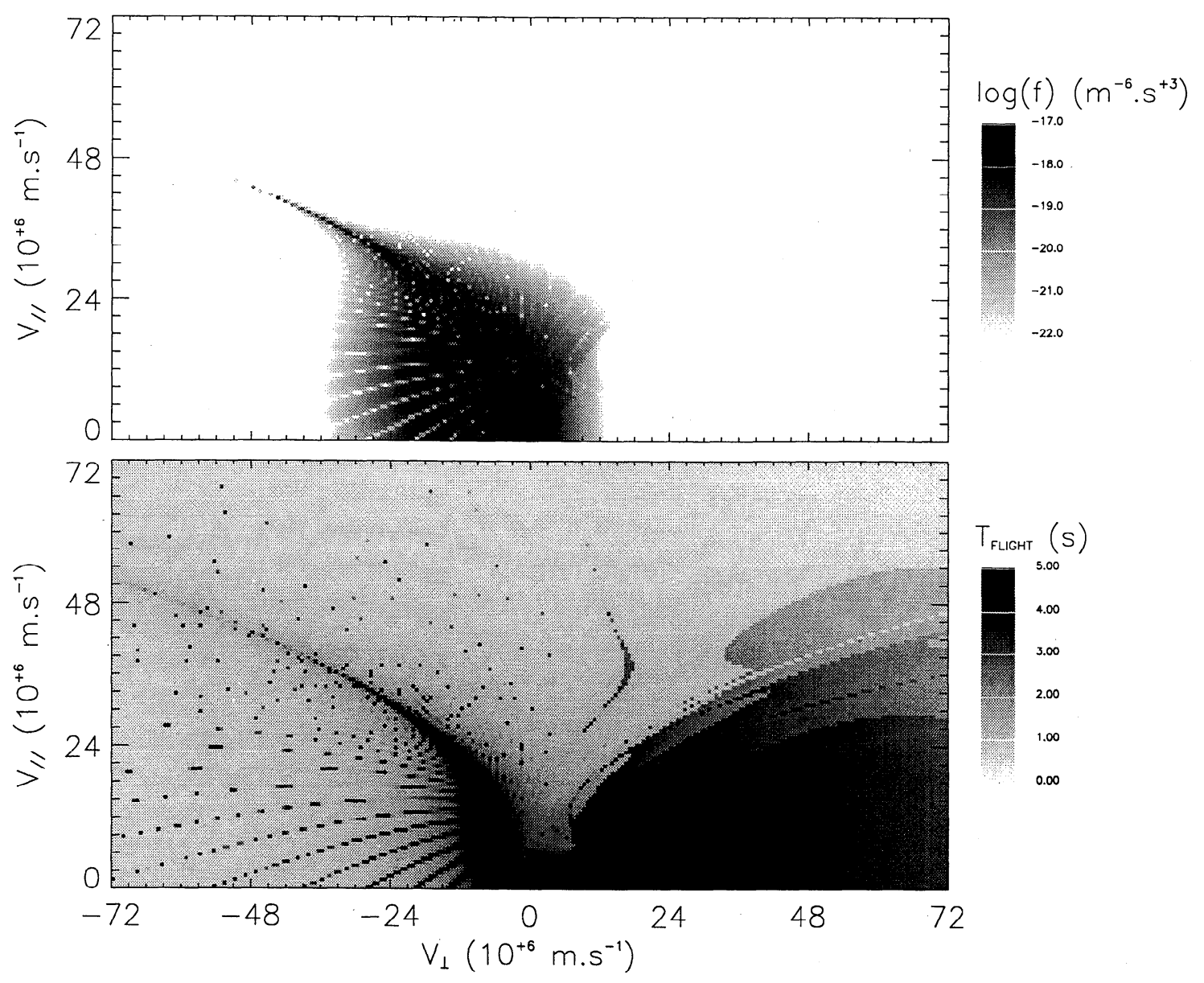

Figure 2. (top) Computed electron distribution function at $x_{o b s}=-1 R_{E}$ and $z_{o b s}=0.01 R_{E}$ and (bottom) electron time of flight from the source location to the observation point. The initial distribution in the magnetotail lobes is taken as a drifting Maxwellian with a temperature of 200 $\mathrm{eV}$ and a bulk flow speed of $400 \mathrm{~km} / \mathrm{s}$.

$\pm 0.6 R_{E}$. As we focus on downstream consequences of nonadiabatic motion in the reconnection region and because of the much larger Larmor radii of ions (hence a larger amplitude of oscillation in the meandering trajectory sequence), we were led to consider an observation point farther away from the neutral line than was found in the case of electrons, namely, $x_{o b s}=-10 R_{E}$ and $z_{o b s}=0.3 R_{E}$ (note here that the computed distribution functions exhibit some variability with distance to the neutral line, an issue we will address in section 6 ). In the top panel of Figure 3 an overall structure similar to that in Figure 2 is observable, that is, two regions of distinct densities separated by a narrow channel with enhanced times of flight (bottom panel). Still, as was mentioned above, the electron motion deviates from adiabaticity in a much smaller $x-z$ interval near the neutral point than that of ions, and so the similarity between Figures 2 and 3 is only qualitative. For example, at a given energy (dotted lines), note the more abrupt transition from one density level to another in the case of ions than that of electrons.

\section{Source Separation and Related Ridge Structure \\ Martin and Speiser [1988] referred to the above twofold} structure of distribution functions near an $\mathrm{X}$ line as a ridge and interpreted them in terms of distinct dynamical regimes. This approach is made more apparent in Figure 4, which presents selected electron trajectories across the ridge structure. It can be seen in this figure that particles that contribute large phase space densities in Figure 2 (Figure 4a) originate from the earthward side of the neutral line (i.e., with pitch angles $\alpha<90^{\circ}$ in the southern hemisphere), whereas particles on the lowdensity side of the ridge (Figure $4 \mathrm{~b}$ ) originate from the distant tail (i.e., $\alpha>90^{\circ}$ in the southern hemisphere). As for the ridge itself (Figure 4c), it corresponds to an intermediate situation where particles are essentially $\mathbf{E} \times \mathbf{B}$ convected from the lobes and significantly accelerated near the neutral point. The negligible parallel speed of these latter particles in the magnetospheric lobes is thus responsible for the large times of flight observed in Figures 2 and 3. 


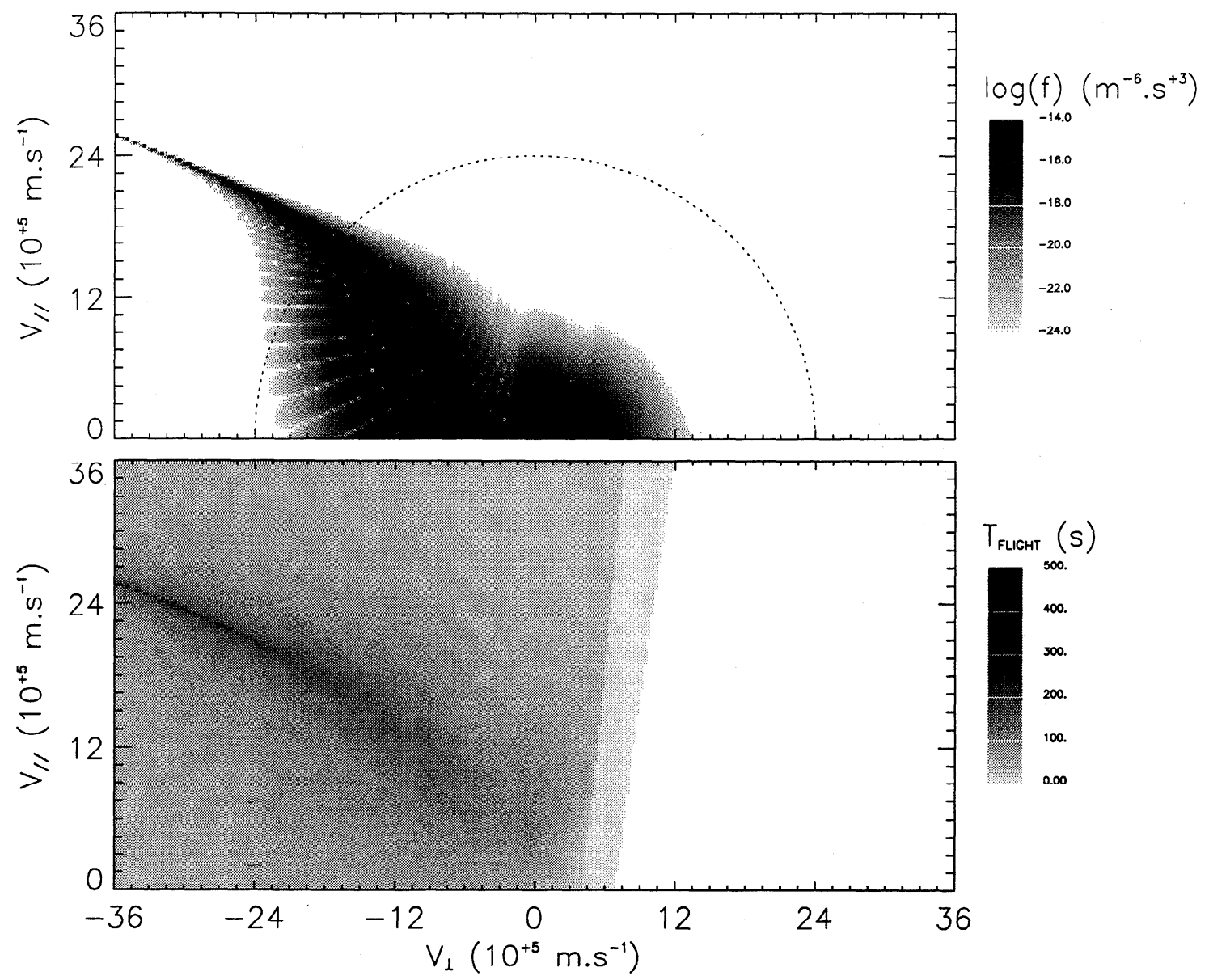

Figure 3. Identical to Figure 2 but for $\mathrm{H}^{+}$ions with a temperature of $400 \mathrm{eV}$. The observation point is placed at $x_{o b s}=-10 R_{E}$ and $z_{o b s}=0.3 R_{E}$.

The top panel of Figure 5 shows the difference between assumed Maxwellian and $\kappa$ distributions at entry versus particle speed. As was mentioned above, a flowing Maxwellian distribution was considered in Figures 2 and 3 , which differs from the flowing $\kappa$ distribution used by Martin and Speiser [1988]. As will be made more apparent in Figure 8, the high-energy tail in the $\kappa$ distribution leads to weaker density gradients in the computed distribution functions, but it does not qualitatively affect their overall structure. This structure is partly due to contributions from distinct velocity space regions. This can be appreciated in the bottom panel of Figure 5, which shows the phase space density at entry as a function of pitch angle (southern hemisphere). It is apparent from this panel that the density gradient near $\alpha=90^{\circ}$ plays a role in the two fold structure portrayed in Figures 2 and 3 . The high-density region in these figures maps into the bulk of the distribution at $\alpha<90^{\circ}$ whereas the low-density one is due to a depleted velocity space at $\alpha>90^{\circ}$. However, this result alone yields density variations about 1 order of magnitude and is insufficient to explain the sharp density gradients observed in the computations. One must consider the energy variations experienced by the particles during transport.
Upon interaction with the neutral line, particles do not conserve their magnetic moment and experience meandering motion about the field minimum; hence their large displacement in the $y$ direction and consequent energization [e.g., Speiser, 1965]. For example, in Figure 4 the tailward originating electron (Figure $4 \mathrm{~b}$ ) experiences an energy variation from $8.2 \mathrm{keV}$ up to $10.2 \mathrm{keV}$, whereas one has a variation from $720 \mathrm{eV}$ up to $10.2 \mathrm{keV}$ for the ridge proper (Figure 4c). These different initial energies for given final energy lead to quite distinct particle weights and thus to prominent density variations in the computed distribution functions. In particular, since the ridge involves particles with relatively low energies which essentially $\mathbf{E} \times \mathbf{B}$ convect from the lobes and experience large energization near the neutral point, this structure is well developed at relatively high energies only (see Figures 2 and 3 ). At lower energies it progressively weakens and merges into the bulk of the distribution function.

\section{Small-Scale Structures}

It is apparent from Figures 2 and 3 that the above description, in terms of two regions of distinct densities separated by a ridge, only accounts for the gross struc- 

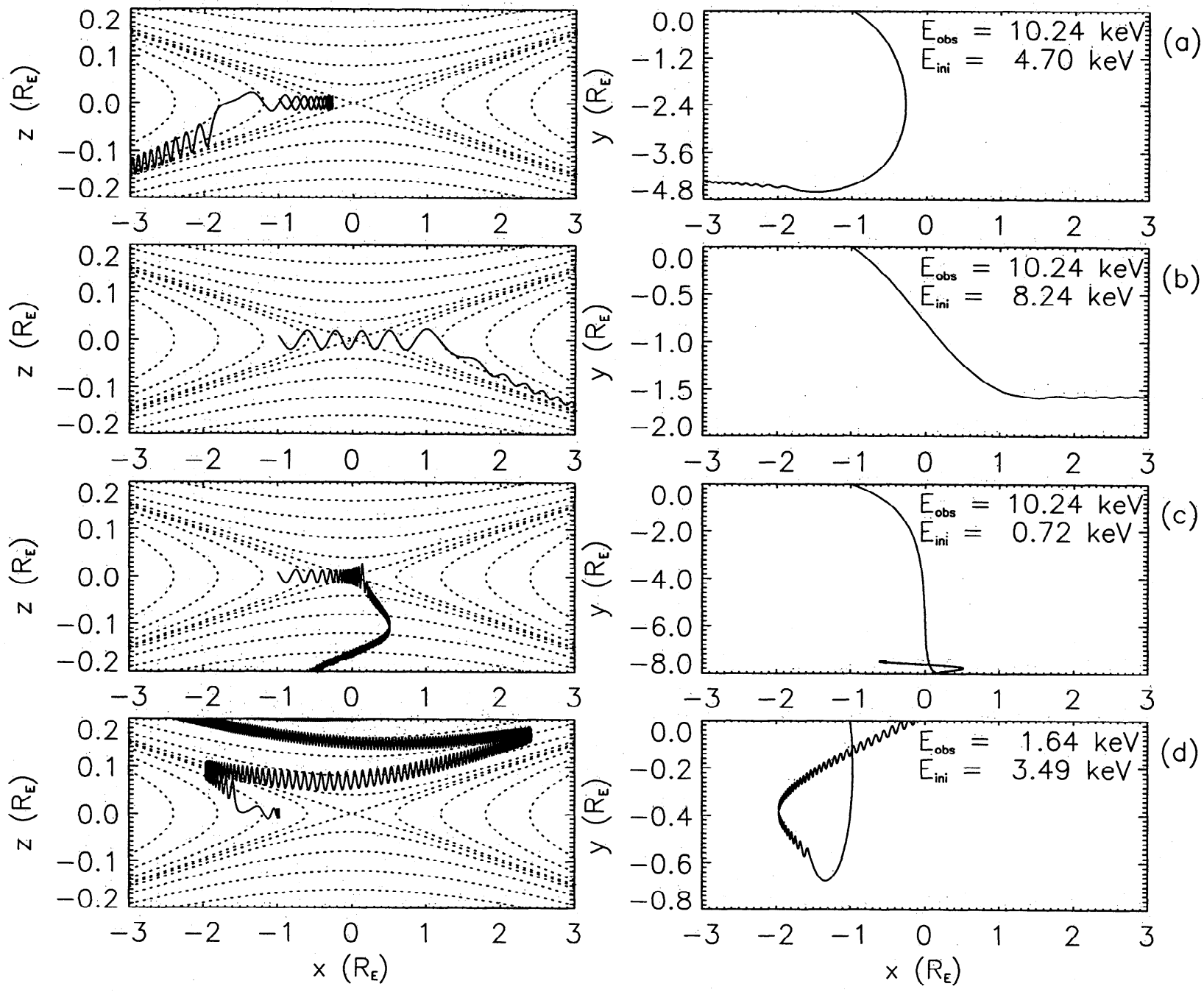

Figure 4. Selected electron trajectories in the (left) $x-z$ and (right) $x-y$ planes. Panels correspond to (a) a test particle contributing to the high-density domain, (b) a particle in the low-density domain, (c) a particle pertaining to the "ridge", and (d) a particle in the "bone-like" structure.
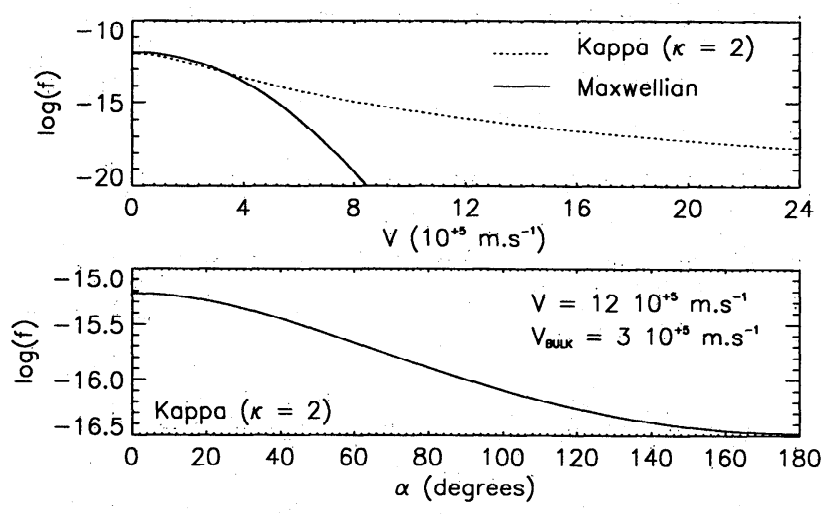

Figure 5. Phase space density versus ion speed assuming either a Maxwellian (solid line) or a $\kappa$ distribution (dotted line) in the source region. Phase space density in the source region as a function of pitch angle (southern hemisphere).

ture of the computed distribution functions. There exist in fact finer "bone-like" structures embedded in each region. The origin of these fine structures can be found by inspection of Figure $4 \mathrm{a}, 4 \mathrm{~b}$, and $4 \mathrm{~d}$ : in the top panels, particles approach the field reversal in a nearly fieldaligned manner and are not subjected to any significant drift in the $y$ direction during this adiabatic sequence. For these particles, prominent $y$ displacement and energization occur only during the meandering trajectory sequence. In contrast, Figure $4 \mathrm{~d}$ shows an electron orbit which contributes to a fine low-density structure. It can be seen that this particle approaches the field reversal in a more perpendicular manner. Because of its large perpendicular momentum, it experiences a two-step energy variation as can be identified from its motion in the $y$ direction (right panel), viz., (1) upon approach, a deceleration due to gradient drift (oriented duskward) and (2) subsequently, a large acceleration during oscillation about the field minimum. In other words, for this particle the energy gained during nonadiabatic motion is 
balanced by that lost during adiabatic transport. The net result is a mapping at a higher energy in the initial distribution and hence a weaker weight as compared to that obtained in the top panels of Figure 4. This behavior was systematically found in the various fine structures of low density (note in this regard the enhanced times of flight associated with these structures in the bottom panels of Figures 2 and 3 , as expected from large pitch angles at the source location).

It is worth noting that these fine structures correspond to distinct $z$ origins for the particles. That is, in a given structure of enhanced density, all the particles originate from the same hemisphere, whereas they originate from the opposite hemisphere in adjacent structures. It should be pointed out that small-scale structuring has been reported in previous studies [e.g., Chen et al., 1990; Burkhart and Chen, 1991] and interpreted as an energy resonance effect whereby particles execute an integer number of $z$ oscillations during gyromotion about the small $b_{z}$. In the present study this effect appears to occur only at specific phases of gyration, and so the relationship between energy resonance and the present results is not straightforward, being complicated by the two-dimensional aspect of the field geometry.

Interestingly; these fine bone structures are absent from the $\mathrm{H}^{+}$distribution functions computed by Martin and Speiser [1988] (see their Figure 3). The reason again lies in the gradient drift that occurs prior to reaching the neutral point. Since electrons turn nonadiabatic only in a small region of space near the neutral point, the preliminary adiabatic sequence extends over a large $z$ interval. As was discussed above, gradient drift during this sequence can give rise to significant energy loss, which in return affects the particle weight. As for ions, they travel adiabatically relatively far away from the neutral point in the magnetospheric lobes and rapidly turn nonadiabatic upon approach of the field reversal. In this case, truncation of the magnetic field model as performed by Martin and Speiser [1988] prevents gradient drift effects prior to interaction with the neutral point. This leads to larger ion energy gains and thus faint bone structures. Because of the adiabatic motion of electrons over a wider $z$ interval inside the neutral sheet, we thus expect small-scale structuring to be more pronounced for these particles.

\section{Distribution Function Variability With View Angle}

In the preceding sections we eluded the variability of the distribution function with view angle at the observation point. This view angle should be important since we are far from an adiabatic description wherein particles are uniformly distributed about a guiding center. In the study of Martin and Speiser [1988] the ridge structure was investigated by averaging over gyration phases. Still, the development of this structure significantly depends upon the direction of view, as is demonstrated in Figure 6 . In this figure, systematic electron trajectory calculations were carried out backward in time to the source location, considering distinct azimuthal angles $\psi$ at the observation point. Here, rather than gyration phase, which is meaningless in the nonadiabatic trajec-
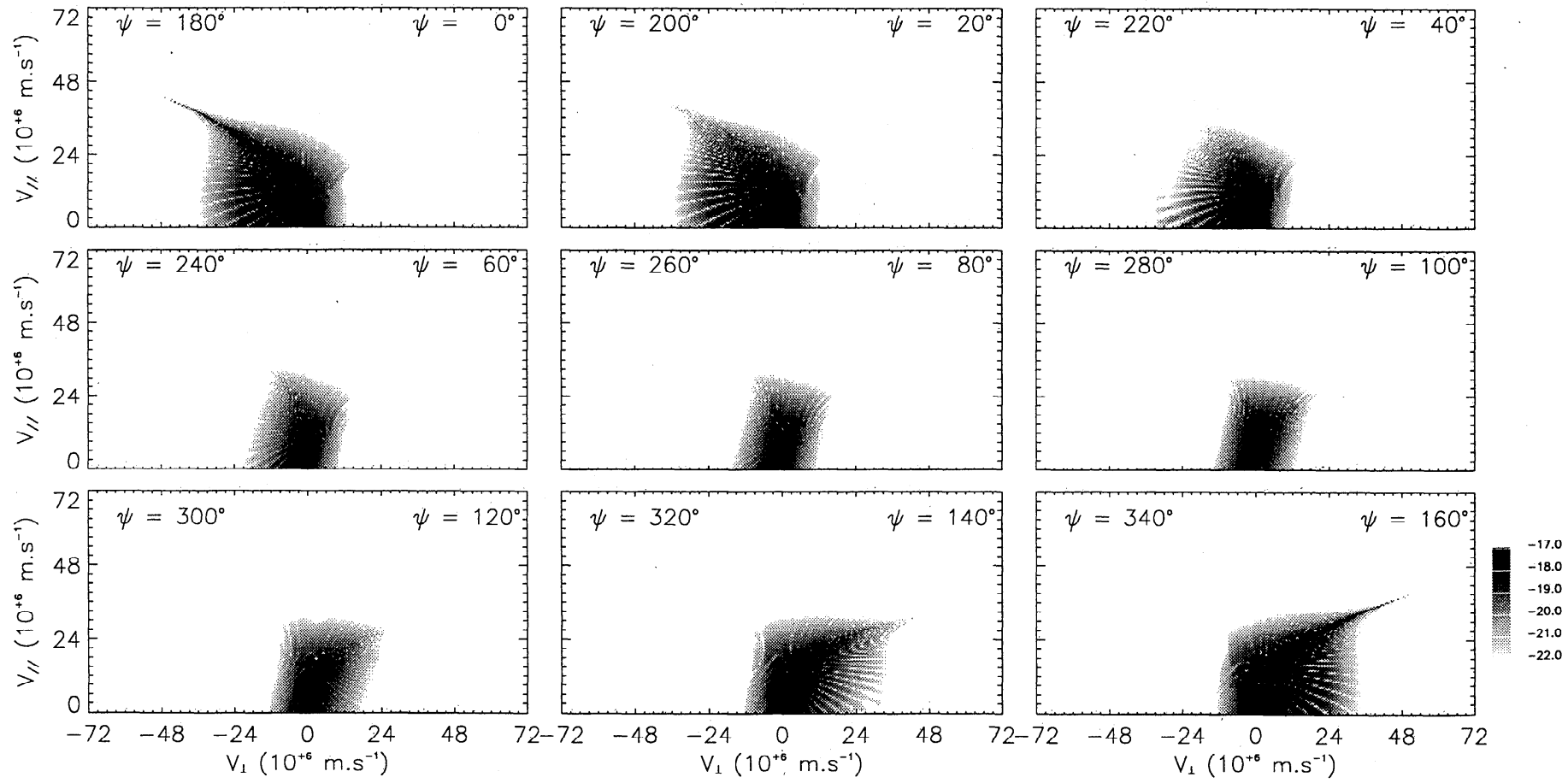

Figure 6. Computed electron distribution function at $x_{o b s}=-1 R_{E}$ and $z_{o b s}=0.01 R_{E}$ for distinct azimuthal view angles (varied by steps of $20^{\circ}$ from upper left panel down to lower right panel). 
tory sequence, we denote by azimuthal angle the angle between the particle velocity component perpendicular to $\mathbf{B}$ and the $y$ axis (in our coordinate system, $\psi=0^{\circ}$ corresponds to a perpendicular particle speed pointing in the $-y$ direction). Each panel of Figure 6 shows the computed pattern obtained at two opposite azimuthal angles (negative and positive $V_{\perp}$ ). Sampling of $\psi$ (by steps of $20^{\circ}$ ) in Figure 6 then provides a full threedimensional view of the electron distribution function at the observation point.

In the upper left panel of Figure 6 one recognizes the pattern displayed in Figure 2. Here, negative $V_{\perp}$ corresponds to $\psi=180^{\circ}$ or, equivalently, to particles leaving the midplane, whereas positive $V_{\perp}\left(\psi=0^{\circ}\right)$ correspond to particles on their way to the midplane. The distinct density domains that are carved out in the three-dimensional velocity space are clearly apparent from Figure 6, namely, (1) a depleted region over most of the azimuthal range (center panels) and (2) a high-density region centered near $\psi=180^{\circ}$ and extending over $40^{\circ}$ in azimuth (upper left and lower right panels). Note also the persistent low-density streaks regardless of view angle.

These results seem to be at variance with those of Speiser and Martin [1992], which display a fairly weak dependence of the ridge structure with gyration phase. This difference is due to the distinct model parameters adopted. Indeed, since our model features a somewhat higher $\mathbf{E} \times \mathbf{B}$ drift velocity, particles may be directly transported from the lobes to the observation point without experiencing meandering motion about the midplane, whereas such a motion will occur for a weaker $\mathbf{E} \times \mathbf{B}$ drift velocity. One can see from this qualitative description that the development of the ridge structure in the full three-dimensional velocity space or only in a restricted sector of it critically depends upon the magnitude of the electric and magnetic fields.

\section{Ridge Characterization}

Martin and Speiser [1988] pointed out a significant dependence of the ridge upon observation position, with a progressive bending toward large pitch angles as the $x$ distance to the neutral point decreases (see their Figure 3). On the other hand, Speiser and Martin [1992] found weak variation with $z$ height away from the midplane. The ridge structure thus implicitly contains information about the locus of reconnection, which could allow remote sensing of the $\mathrm{X}$ line. In a subsequent study, Speiser and Martin [1995] developed this approach to model distribution functions in the vicinity of an assumed near-Earth neutral line during a substorm and reproduced with some success AMPTE/IRM observations.

To further characterize the ridge, we can take advantage of the fact that particles that contribute to it are essentially $\mathbf{E} \times \mathbf{B}$ convected from the lobes and subsequently accelerated near the neutral point. If we consider the distribution function obtained for $\psi=180^{\circ}$

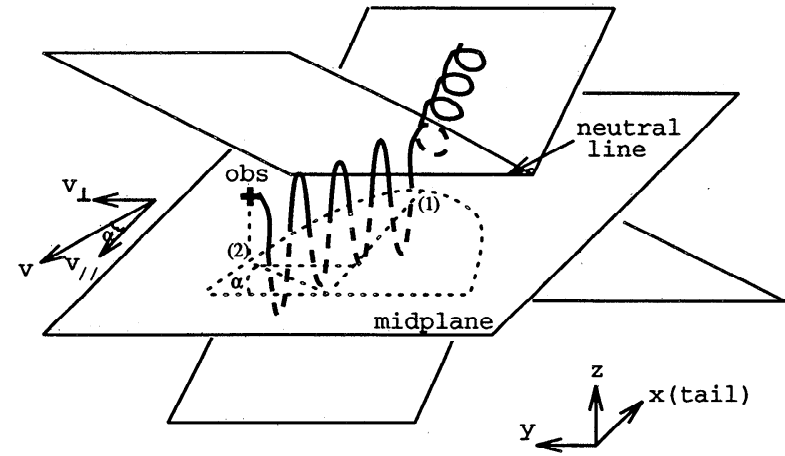

Figure 7. Schematic perspective plot of a particle orbit near the neutral line. The cross shows the observation point.

(upper left panel in Figure 6), it corresponds in our coordinate system to outbound particles near the end of the meandering trajectory sequence. Moreover; as is schematically illustrated in Figure 7, we note that particles in the nearly perpendicular direction (labeled 1 in Figure 7) have executed about one fourth of a cyclotron turn about the small $b_{z}$ component, whereas particles in the nearly parallel direction (labeled 2 in Figure 7) have performed about half a turn about the small $b_{z}$. In view of Figure 4, we approximate the ridge particles as those that cross the separatrix $z^{2}-\delta \times x^{2}=0$ near the beginning of the meandering trajectory sequence, i.e., those that have a velocity nearly pointing in the $y$ direction at this point. During meandering, the $z$ equation of motion $\ddot{z}=-\left(q b_{o} / m\right) \dot{y} z$ with a nearly constant $\dot{y}$ yields oscillations within two limiting values of $\simeq z_{l i m}$, whereas displacement in $x$ depends upon the Larmor radius $m V \sin \alpha / q b_{z}$. One then obtains for the closest position to the neutral point (located at the axis origin) during meandering

$$
\begin{aligned}
x & =\zeta_{x} x_{o b s}+\frac{m V}{q b_{o} \delta \zeta_{x} x_{o b s}}(1-\sin \alpha) \\
z & =z_{l i m} \sim z_{o b s}
\end{aligned}
$$

Here, $x_{o b s}$ and $z_{o b s}$ are the coordinates of the observation point, $m$ is the particle mass, $q$ is its charge, and $\alpha$ is its pitch angle (at the observation point). Several approximations have been made in deriving (2), such as neglecting energy and $b_{z}$ variations during meandering. These approximations, which we validate a posteriori by comparison with the computations, are gathered within the $\zeta_{x}$ coefficient, which we derive from a fit to the numerical results. As can be seen in Table 1, this fit leads

Table 1. Values of the $\zeta_{x}$ Coefficient in equation (2) for the Distinct Cases Considered in Figures 8 and 9

\begin{tabular}{cll}
\hline Figure & Particle Species & $\zeta_{x}$ \\
\hline & & \\
8 & $\mathrm{H}^{+}$ & 0.44 \\
$9 \mathrm{a}$ & $e^{-}$ & 0.6 \\
$9 \mathrm{~b}$ & $e^{-}$ & 0.56 \\
$9 \mathrm{c}$ & $e^{-}$ & 0.48 \\
\hline
\end{tabular}




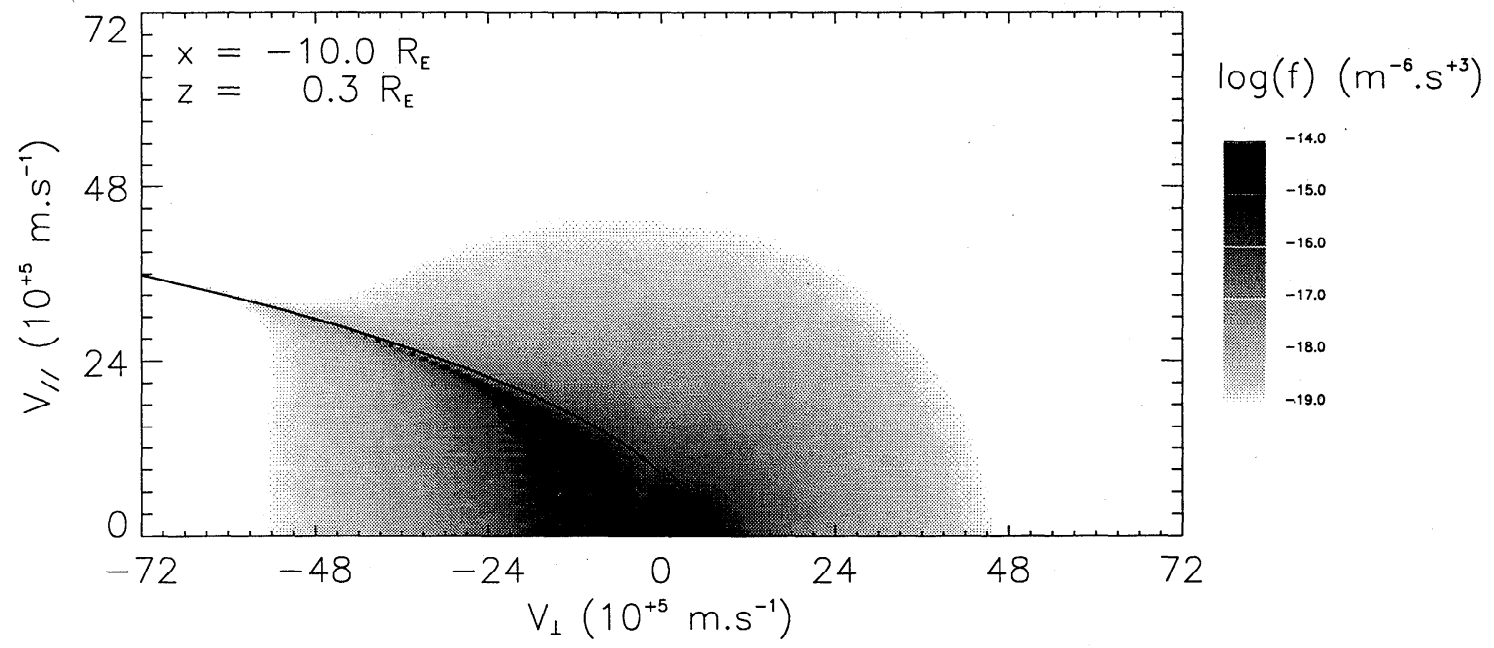

Figure 8. Identical to Figure 3 but using a $\kappa$ distribution at entry. The heavy solid line depicts the ridge analytical location obtained from (2) and (3).

with a good accuracy to $\zeta_{x}$ of the order of 0.5 . This values amounts, for instance, to considering the average $b_{z}$ component between observation and neutral points. At the observation point, particles pertaining to the ridge will thus have a velocity $V$ and a pitch angle $\alpha$ that satisfy equations (2) and (3) with the additional constraint $z^{2}-\delta \times x^{2}=0$ between (2) and (3).

Figure 8 shows how this analytical estimate of the ridge location compares with numerical results. In this figure, similarly to Martin and Speiser [1988], we have considered $\mathrm{H}^{+}$ions with a flowing $\kappa$ distribution in the magnetospheric lobes. The computed distribution in Figure 8 clearly resembles that in Figure 3 of Martin and Speiser [1988]. Moreover, we have calculated from (2)-(3) the set of $V$ and $\alpha$ values that we expect to correspond to the ridge. It is apparent from Figure 8 that a good agreement is obtained between analytical and numerical locations of the ridge (as is indicated in Table 1; note that we used here $\zeta_{x}=0.44$ in (2)). In particular, the analytical estimate (heavy solid line) re- produces well the progressive bending of the ridge as the $\mathrm{H}^{+}$energy increases. We pursued this analysis further by examining the ridge variation with $x$ distance to the neutral point. It is easy to see from (2) that, given the particle velocity $V$, decreasing $x$ implies increasing $\alpha$. In other words, one expects from (2) a shift of the ridge structure toward large pitch angles as the $x$.distance decreases. This effect is illustrated in Figure 9, which shows electron distribution functions at selected $x$ distances from the neutral point. It is apparent from this figure that the present analytical estimate nearly coincides with the numerical results, exhibiting a ridge at larger pitch angles closer to the neutral point as put forward in the analysis of Martin and Speiser [1988]. Note here again in Table 1 that the coefficient $\zeta_{x}$ in the analytical estimate (2) is of the order of 0.5 .

Finally, it should be kept in mind that the ridge structure is limited to the close vicinity of the reconnection line, since away from it, particles may experience multiple crossings of the field reversal which leads to a more

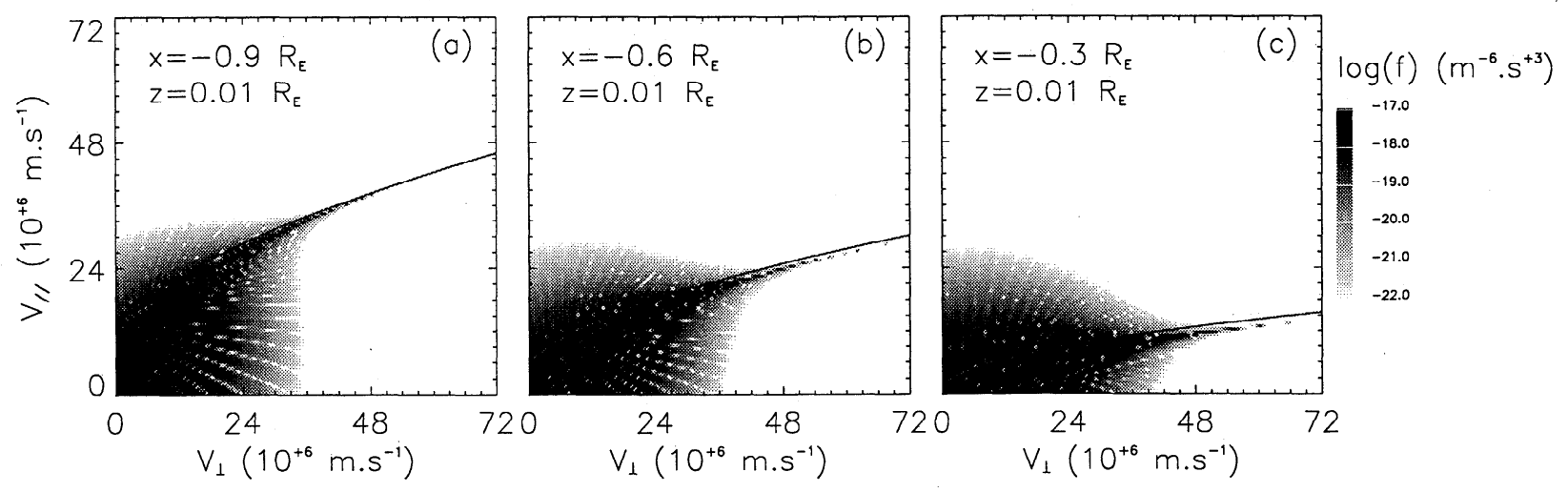

Figure 9. Identical to Figure 2 but for distinct $\mathrm{X}$ distances to the neutral point: (a) $-0.9 R_{E}$, (b) $-0.6 R_{E}$ and (c) $-0.3 R_{E}$. In each panel, the heavy solid line depicts the ridge analytical location obtained from (2) and (3). 
complex picture. Still, knowledge of $V$ and $\alpha$ values along an observed ridge provides information on the magnetic field topology, since one has from (2)

$$
\delta b_{o} x_{o b s}\left(\frac{z_{o b s}}{\sqrt{\delta}}-x_{o b s}\right)=\frac{m V}{q}(1-\sin \alpha)
$$

With the additional conditions $b_{o} z_{o b s}=b_{x}$ and $b_{o} \delta x_{o b s}=$ $b_{z}$ (where $b_{x}$ and $b_{z}$ are the magnetic field components measured at the observation point), we obtain three equations relating $x_{o b s}, z_{o b s}, b_{o}$, and $\delta$. Though underdetermined as such, this system of equations allows determination of $x_{o b s}, z_{o b s}$, and $\delta$ with assumed value of the asymptotic field $b_{o}$ in the magnetospheric lobes.

\section{Conclusion}

Model calculations of electron and ion trajectories in the vicinity of an $\mathrm{X}$ line in the magnetotail reveal that downstream distribution functions are divided into distinct density domains. This overall structure, which was first reported for ions by Martin and Speiser [1988], weakly depends upon the characteristics of the inflowing populations. It results from mixing of particles originating earthward and tailward of the neutral line. The trajectory calculations also reveal multiple small-scale structures, due to prominent gradient drift effects prior to crossing of the field reversal, which may represent a useful electron signature of the neutral line. As a result of nonadiabatic motion the downstream distribution functions exhibit a pronounced azimuthal asymmetry with a well-developed ridge in a narrow sector of view angles. This ridge structure provides information on the reconnection site, since it corresponds to an intermediate regime where low-energy particles $\mathbf{E} \times \mathbf{B}$ convecting from the lobes are accelerated along the neutral line.

Acknowledgmonts. The Editor thanks G. M. Erickson and two other referees for their assistance in evaluating this paper.

\section{References}

Büchner, J., and L. M. Zelenyi, Regular and chaotic charged particle motion in magnetotaillike field reversals: 1 . Basic theory of trapped motion, J. Geophys. Res. 94, 11,821, 1989.

Burkhart, G. R., and J. Chen, Differential memory in the Earth's magnetotail, J. Geophys. Res. 96, 14,033, 1991.

Burkhart, G. R., R. F. Martin Jr., P. B. Dusenbery, and T. W. Speiser, Neutral line chaos and phase space structure, Geophys. Res. Lett. 18, 1591, 1991.

Chen, J., and P. J. Palmadesso, Chaos and nonlinear dynamics of single-particle orbits in magnetotaillike magnetic field, J. Geophys. Res. 91, 1499, 1986.

Chen, J., G. R. Burkhart, and C. Y. Huang, Observational signature of nonlinear magnetotail particle dynamics, Geophys. Res. Lett. 17, 2237, 1990.

Chen, J., Nonlinear dynamics of charged particles in the magnetotail, J. Geophys. Res. 97, 15,011, 1992.
Curran, D. B., and C. K. Goertz, Particle distributions in a two-dimensional reconnection field geometry, J. Geophys. Rcs. 94, 272, 1989.

Delcourt, D. C., J.-A. Sauvaud, R. F. Martin Jr., and T. E. Moore, Gyrophase effects in the centrifugal impulse model of particle motion of the magnetotail, J. Geophys. Res. 100, 17,211, 1995.

Doxas, I., T. W. Speiser, P. B. Dusenbery, and W. Horton, A proposed neutral line signature, J. Geophys. Res. 99, 2375, 1994.

Joyce, G., J. Chen, S. Slinker, D. L. Holland, and J. B. Harold, Particle energization near an X line in the magnetotail based on global MHD fields, J. Geophys. Res. 100, 19,167, 1995.

Lyons, L. R., Electron energization in the geomagnetic tail current sheet, J. Geophys. Res. 89, 5479, 1984.

Martin, R. F., Jr., Chaotic particle dynamics near a twodimensional magnetic neutral point with application to the geomagnetic tail, J. Geophys. Res. 91, 11,985, 1986.

Martin, R. F., Jr., and T. W. Speiser, A predicted energetic ion signature of a neutral line in the geomagnetic tail, $J$. Geophys. Res. 93, 11,521, 1988.

Onsager, T. G., M. F. Thomsen, R. C. Elphic, and J. T. Gosling, Model of electron and ion distributions in the plasma sheet boundary layer, J. Geophys. Res. 96, 20,999, 1991.

Schriver, D., and M. Ashour-Abdalla, Signatures of electron acceleration in the magnetotail, in Physics of Space: Plasmas, SPI Conference Proceedings, edited by T. Chang, (Scientific Publishers. inc., Cambridge, MA), 1993.

Speiser, T. W., Particle trajectory in model current sheets, 1, Analytical solutions, J. Geophys. Res. 70, 4219, 1965.

Speiser, T. W., and R. F. Martin Jr., Energetic ions as remote probes of $\mathrm{X}$ type neutral lines in the geomagnetic tail, J. Geophys. Res. 97, 10,775, 1992.

Speiser, T. W., and R. F. Martin Jr., Neutral line energetic ion signatures in the geomagnetic tail: Comparisons with AMPTE observations, in Space Plasmas:Coupling Between Small and Medium Scale Processes, Geophys. Monogr. ser., vol. 86, edited by M. Ashour-Abdalla, T. Chang, and P. Dusenbery, p. 243, (AGU, Washington, D. C.), 1995.

Tsyganenko, N. A., A magnetospheric magnetic field model with a warped tail current sheet, Planet. Space Sci., 37, 5, 1989.

'elenyi, L. M., R. A. Kovrazhkin, and J.-M. Bosqued, Velocity-dispersed ion beams in the nightside auroral zone: AUREOL-3 observations, J. Geophys. Res. 95, 12,119, 1990.

D. Delcourt, R. Smets, Centre d'étude des Environnements Terrestre et Planétaires, Centre National de la Recherche Scientifique, 4 avenue de Neptune, 94107 SaintMaur des Fossés, France. (e-mail: delcourt@cetp.ipsl.fr, and smets@cetp.ipsl.fr)

D. Fontaine, Centre d'étude des Environnements Terrestre et Planétaires, Centre National de la Recherche Scientifique, 10-12 avenue de l' Europe, 78140 Vélizy, France. (e-mail: fontaine@cetp.ipsl.fr)

R. F. Martin, Jr., Physics Department, Illinois State University, Normal, IL 61761 (e-mail: rfm@entropy.phy.ilstu. edu)

(Received February 13, 1996; revised July 10, 1996; accepted July 11, 1996.) 